\title{
SNAP satellite focal plane development
}

\section{Christopher Bebek, Carl W. Akerlof, Greg Aldering, R. Amanullah, Pierre Astier, et al.}

Christopher Bebek, Carl W. Akerlof, Greg Aldering, R. Amanullah, Pierre Astier, Charles Baltay, E. Barrelet, Stephane Basa, John Bercovitz, Lars Bergstrom, Gary Berstein, Manfred Bester, Ralph C. Bohlin, Alain Bonissent, Charles R. Bower, Myron Campbell, William C. Carithers, Eugene D. Commins, C. Day, Susana E. Deustua, Richard S. DiGennaro, Anne Ealet, Richard S. Ellis, William Emmett, Mikael Eriksson, D. Fouchez, Andrew Fruchter, Jean-Francois Genat, Gerson Goldhaber, Ariel Goobar, Donald E. Groom, Henry D. Heetderks, Stephen E. Holland, Dragan Huterer, William E. Johnston, Richard W. Kadel, Armin Karcher, Alex G. Kim, William F. Kolbe, Robin E. Lafever, J. I. Lamoureux, Michael L. Lampton, Oliver LeFevre, Michael E. Levi, Daniel S. Levin, Eric V. Linder, Stewart C. Loken, Roger Malina, Alain Mazure, Timothy A. McKay, Shawn P. McKee, Ramon Miquel, Nicholas Morgan, E. Mortsell, N. Mostek, Stuart Mufson, J. A. Musser, Natalie A. Roe, Peter E. Nugent, Hakeem M. Oluseyi, Reynald Pain, Nicholas P. Palaio, David H. Pankow, Saul Perlmutter, Eric Prieto, David Rabinowitz, Alexandre Refregier, Jason Rhodes, Michael S. Schubnell, Michael Sholl, G. Smadja, R. Smith, George F. Smoot, Jeffrey A. Snyder, Anthony Spadafora, Andrew Szymkowiak, Gregory Tarle, Keith Taylor, A. Tilquin, Andrew D. Tomasch, D. Vincent, Henrik von der Lippe, JeanPierre Walder, Guobin Wang, "SNAP satellite focal plane development," Proc. SPIE 5164, UV/EUV and Visible Space Instrumentation for Astronomy II, (8 December 2003); doi: 10.1117/12.505232 


\title{
SNAP Satellite Focal Plane Development
}

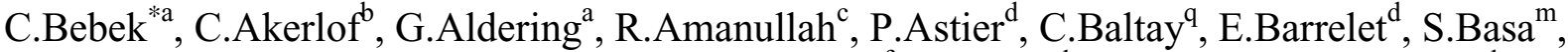 \\ J.Bercovitz $^{\mathrm{a}}$, L.Bergstrom ${ }^{\mathrm{c}}$, G.Berstein ${ }^{\mathrm{e}}$, M.Bester ${ }^{\mathrm{f}}$, R.Bohlin $^{\mathrm{k}}$, A.Bonissent ${ }^{\mathrm{m}}$, C.Bower ${ }^{\mathrm{h}}$,

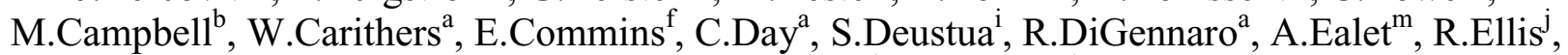 \\ W.Emmett $^{\mathrm{q}}$, M.Eriksson ${ }^{\mathrm{c}}$, D.Fouchez ${ }^{\mathrm{m}}$, A.Fruchter ${ }^{\mathrm{k}}$, J-F.Genat ${ }^{\mathrm{d}}$, G.Goldhaber ${ }^{\mathrm{a}}$, A.Goobar ${ }^{\mathrm{c}}$,

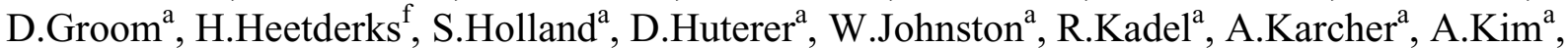 \\ W.Kolbe ${ }^{a}$, R.Lafever ${ }^{a}$, J.Lamoureaux ${ }^{a}$, M.Lampton ${ }^{f}$, O.Lefevre ${ }^{a}$, M.Levi ${ }^{a}$, D.Levin ${ }^{b}$, E.Linder ${ }^{a}$, \\ S.Loken $^{a}$, R.Malina ${ }^{\mathrm{m}}$, A.Mazure ${ }^{\mathrm{m}}$, T.McKay ${ }^{\mathrm{b}}$, S.McKee $^{\mathrm{b}}$, R.Miquel ${ }^{\mathrm{a}}$, N.Morgan ${ }^{\mathrm{q}}$, E.Mortsell $^{\mathrm{c}}$, \\ N.Mostek ${ }^{h}$, S.Mufson ${ }^{\text {h }}$, J.Musser ${ }^{\mathrm{h}}$, N.Roe ${ }^{\mathrm{a}}$, P.Nugent ${ }^{\mathrm{a}}$, H.Oluseyi $^{\mathrm{a}}$, R.Pain ${ }^{\mathrm{d}}$, N.Palaio ${ }^{\mathrm{a}}$, D.Pankow ${ }^{\mathrm{f}}$, \\ S.Perlmutter $^{\mathrm{a}}$, E.Prieto ${ }^{\mathrm{m}}$, D.Rabinowitz ${ }^{\mathrm{q}}$, A.Refregier ${ }^{\mathrm{j}}$, J.Rhodes $^{\mathrm{j}}$, M.Schubnell $^{\mathrm{b}}$, M.Sholl ${ }^{\mathrm{f}}$, \\ G.Smadja $^{\mathrm{m}}$, R.Smith ${ }^{\mathrm{j}}$, G.Smoot ${ }^{\mathrm{a}}$, J.Snyder ${ }^{\mathrm{q}}$, A.Spadafora ${ }^{\mathrm{a}}$, A.Szymkowiak ${ }^{\mathrm{q}}$, G.Tarle ${ }^{\mathrm{b}}$, K.Taylor ${ }^{\mathrm{j}}$,

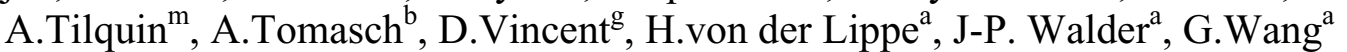 \\ ${ }^{a}$ Lawrence Berkeley National Laboratory \\ ${ }^{\mathrm{b}}$ University of Michigan, Ann Arbor MI, USA \\ ${ }^{\mathrm{C}}$ University of Stockholm, Stockholm, Sweden \\ ${ }^{\mathrm{d}} \mathrm{CNRS} / \mathrm{IN} 2 \mathrm{P} 3 / \mathrm{LPNHE}$, Paris, France \\ ${ }^{\mathrm{e}}$ University of Pennsylvania, Philadelphia, PA, USA \\ ${ }^{f}$ University of California, Berkeley, CA, USA \\ ${ }^{\mathrm{g}} \mathrm{CNRS} / \mathrm{IN} 2 \mathrm{P} 3 / \mathrm{CPPM}$, Marseille, France \\ ${ }^{\mathrm{h}}$ Indiana University, Bloomington, IN, USA \\ ${ }^{i}$ American Astronomical Society, Washington, DC, USA \\ ${ }^{\mathrm{j}}$ California Institute of Technology, Pasadena, CA, USA \\ ${ }^{\mathrm{k}}$ Space Telescope Science Institute, Baltimore, MD, USA \\ ${ }^{1}$ Case Western Reserve University, Cleveland, OH,USA \\ ${ }^{\mathrm{m}} \mathrm{CNRS} / \mathrm{INSU} / \mathrm{LAM}$, Marseille, France \\ ${ }^{\mathrm{n}}$ Cambridge University, Cambridge, UK \\ ${ }^{\circ}$ NASA Goddard Space Flight Center, Greenbelt, MD, USA \\ ${ }^{\mathrm{p}} \mathrm{CNRS} / \mathrm{IN} 2 \mathrm{PL}$, Lyon, France \\ ${ }^{q}$ Yale University, New Haven, CN, USA
}

\begin{abstract}
The proposed SuperNova/Acceleration Probe (SNAP) mission will have a two-meter class telescope delivering diffraction-limited images to an instrumented 0.7 square degree field in the visible and near-infrared wavelength regime. The requirements for the instrument suite and the present configuration of the focal plane concept are presented. A two year R\&D phase, largely supported by the Department of Energy, is just beginning. We describe the development activities that are taking place to advance our preparedness for mission proposal in the areas of detectors and electronics.
\end{abstract}

Keywords: ASIC, CCD, optical filters, focal plane detectors, $\mathrm{HgCdTe}$, InGaAs, spectrograph edited by Oswald H. W. Siegmund, (SPIE, Bellingham, WA, 2003) $\cdot 0277-786 X / 03 / \$ 15.00$ 


\section{INTRODUCTION}

The SuperNova/Acceleration Probe (SNAP) is a planned space flight mission intended to obtain a precise determination of the expansion history of the universe[1]. The experiment is prompted by the discovery[2,3] that this expansion is accelerating, not decelerating as would be predicted by models that contain mass-energy but no cosmological constant or dark energy. SNAP redshift and magnitude data of several thousand standardizable Type Ia supernovae ( $\mathrm{SNe}$ ) can be compared to model predictions of the expansion rate as a function of lookback time, or equivalently, luminosity distance. Each measured supernova furnishes a redshift and an effective peak magnitude. The supernova magnitude is a measure of the distance to the supernova, and hence the elapsed time since the supernova exploded. Redshift is a measure of the expansion between its epoch and the present. A description of the mission and its science is presented in the Mission Definition and Requirements Document at http://snap.lbl.gov. SNAP science goals place the following high level requirements on the instrument:

- Early detection of supernovae.

- B-band rest-frame photometry to follow a supernova light curve.

- Supernova color at peak and near-peak brightness.

- Photometric redshifts of the host galaxies prior to supernova follow-up.

- Optical and near infrared spectra at peak brightness to classify the supernovae.

- Optical and near infrared spectra for more precise host galaxy z determination..

- Medium resolution spectra for a limited subset of supernovae over the full light-curve.

\section{INSTRUMENT CONCEPT}

The SNAP science program requires the discovery and detailed follow-up observations of at least 2000 Type Ia supernovae with redshifts ranging from 0.3 to 1.7 . The photometric data are sent to a ground station and analyzed every few days in order to schedule a spectroscopic measurement near peak luminosity for candidate Type Ia SNe. Additionally, a few $\mathrm{SNe}$ with $\mathrm{z}<0.7$ will have spectra taken at several stages of their evolution to develop a set of spectral templates.

A 2-m three mirror anastigmat telescope, diffraction limited at $1 \mu \mathrm{m}$ over a $1.7 \mathrm{deg}^{2}$ field of view, illuminates a 500 $\mathrm{mm}$ diameter focal plane[4]. Discovery and photometric follow-up are automatically accomplished with the large field of view imager that repetitively scans a fixed region of sky. A spectrometer optimized for observing SN spectra is allocated observation time for follow-up spectroscopy and template building. To meet the above requirements, SNAP has a large, $0.7 \mathrm{deg}$. ${ }^{2}$ instrumented field of view and an observation cadence of 4 days, commensurate with SN rise and fall times.

Figure 1 shows critical points on the light curve and the desired measurement accuracy. We note that the required signal-to-noise ratio $(\mathrm{S} / \mathrm{N})$ need not be achieved with a single measurement but can be synthesized from multiple measurements, taking advantage of the substantial time dilation for high-redshift SNe. A typical SN spectrum at peak brightness is shown in Figure 2. The restframe UV side of the spectrum extends down to $350 \mathrm{~nm}$ for $\mathrm{z}=0.3$ while the signature SiII line is at $1700 \mathrm{~nm}$ for $\mathrm{z}=1.7$. A very modest resolution $\lambda / \delta \lambda$ of 100 is sufficient to measure the spectral features in Figure 2.

The SNAP focal plane concept and operation have remained fairly stable since last reported[5]. A wide-field visible and NIR imager, an integral field unit spectrograph, and star guiders are located on a common cold plate passively cooled to $140 \mathrm{~K}$. The reference design uses LBNL CCDs for visible wavelengths and HgCdTe for the NIR. A fixed array of filters over the imager sensors is used for multi-band photometry. 


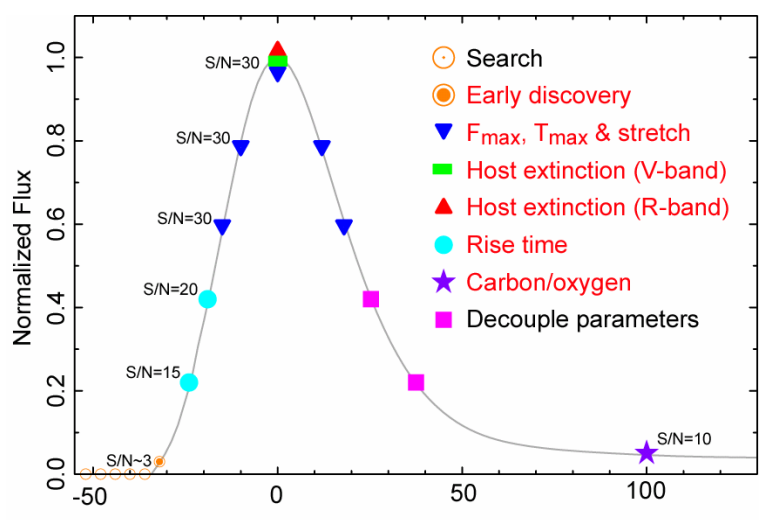

Days from Maximum (Observer Frame)

Figure 1. Sample SN restframe B-band light curve for a $\mathrm{z}=0.8$ Type Ia supernova. Required $\mathrm{S} / \mathrm{N}$ at different epochs are shown.

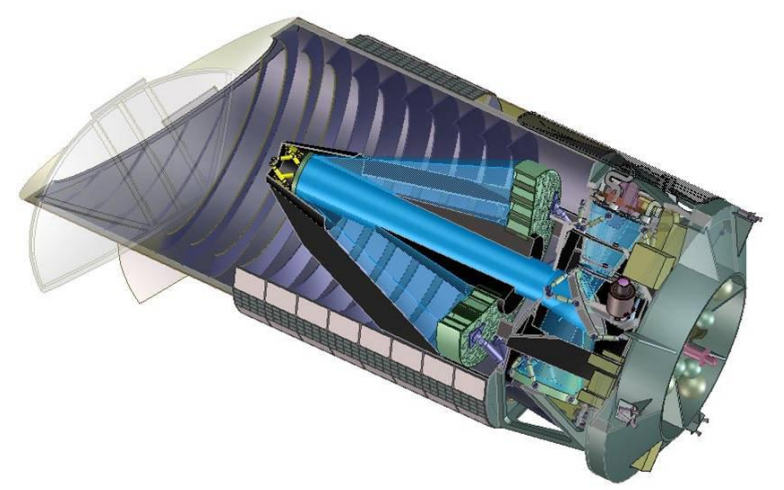

Figure 3. SNAP satellite. The instrument focal plane in at the upper right.

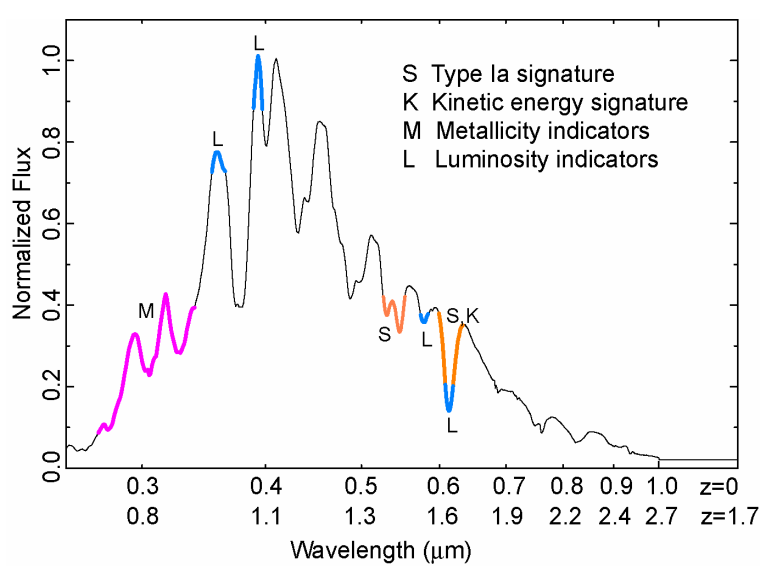

Figure 2. Type Ia spectrum at maximum brightness showing critical features. The horizontal axis shows wavelengths for $\mathrm{z}=0$ and $\mathrm{z}=1.7$.

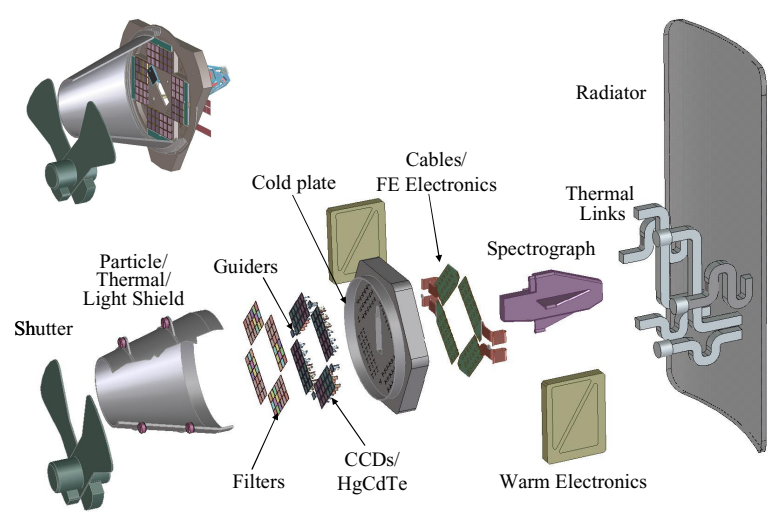

Figure 4. Exploded view of the focal plane region. Shown is an imager using CCDs and $\mathrm{HgCdTe}$ sensors and discrete filters.

A simple example of a fixed-filter focal plane is shown in Figure 5. A linear array of sensors with different filters is swept across a star field by steering the satellite. The actual motion is a series of steps, each step having a fixed exposure time. The number of steps within a filter determines the total integration time. Note that multiple steps within a filter facilitate standard techniques for optimizing photometric accuracy such as dithering. For example, four exposures within a filter could be offset precisely from each other by a fraction of a pixel size. A bigger hop to the next filter then would follow.

In this simple example, some filters are longer than others so as to gain longer integration time in the red and NIR, where SN signals are weaker. To minimize scan start and stop overheads, this scan strategy favors long, linear scan regions of the sky.

To perform a scan of fixed sky region repetitively with all stars passing over the same set of filter bandpasses, the satellite and

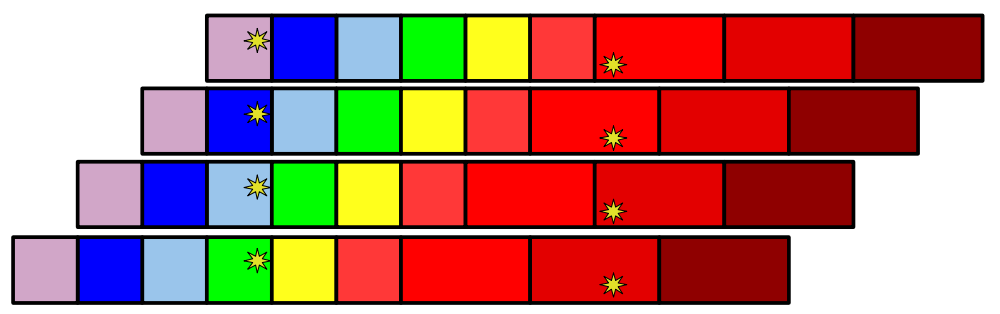

Figure 5. A simple example depicting a linear array of fixed filters. Shown are the positions of a single star as the filters are dragged past it. focal plane is held in fixed alignment relative to the observation field for three month periods after which the satellite is rotated $90^{\circ}$. This maneuver imposes the requirement of a two- rather a one-dimension deployment of filters, as shown in Figure 6. Even though SNAP has 
fixed, body mounted solar cells and a passive heat radiator, the satellite orientation relative to normal solar incidence can tolerate rotations up to $\pm 45^{\circ}$. The two-dimensional symmetry of the filter deployment allows continued scanning through the same set of filter bands, albeit physically different ones, after satellite rotation.

The NIR sensor physical size is fixed by the vendor. We have control of the CCD size, so that it is possible to make the physical dimensions of the $6 \times 6$ CCD filter array equal to those of the $3 \times 3$ NIR filter array. Underlying each NIR filter is one $2 \mathrm{k} \mathrm{x} 2 \mathrm{k}, 18 \mu \mathrm{m} \mathrm{HgCdTe}$ device, 36 in total. Underlying each $2 \times 2$ array of visible filters is one $3.5 \mathrm{k} \times 3.5 \mathrm{k}, 10.5$ $\mu \mathrm{m} \mathrm{CCD}$, also 36 in total.

Also, shown in Figure 6 is the spectrograph and its port. After ground-based trigger, a supernova near peak brightness is guided onto the port by steering the satellite. The last item shown is a collection of sensors that can provide focal plane guiding information to the spacecraft. The imager, spectrograph, and star guiders are discussed in more detail below.

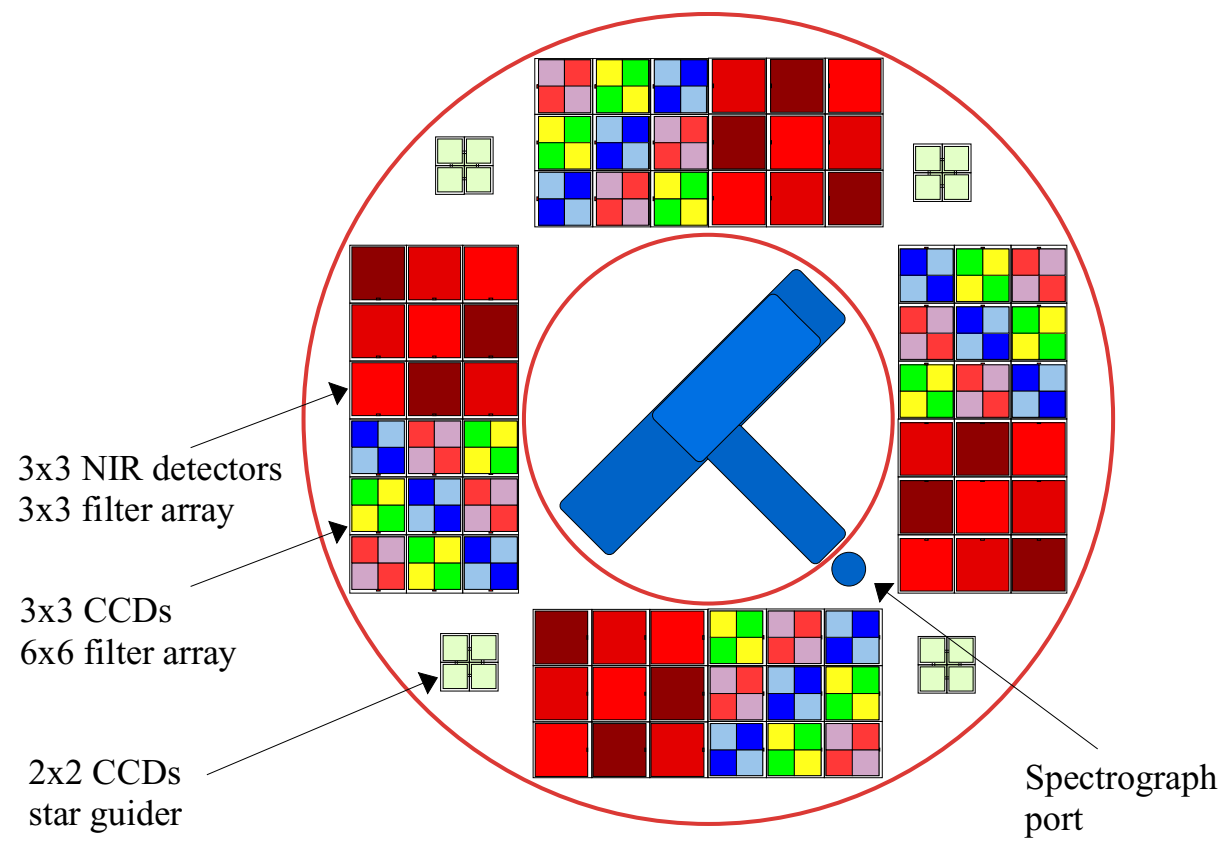

Figure 6. The SNAP focal plane working design. The two axis symmetry of the imager filters allows any $90^{\circ}$ rotation to scan a fixed strip of the sky and measure all objects in all nine filter types. The central rectangle and solid circle are the spectrograph body and its light access port, respectively. The spectrum of a supernova is taken by placing the star in the spectrograph port by steering the satellite. The four small, isolated squares are the star guider CCDs. The inner and outer radii are 129 and $284 \mathrm{~mm}$, respectively.

\section{IMAGER}

The photometry requirements on the imager are (refer to Figure 1):

- Measurement of rest frame U, B, V, (R)-band light curves.

- Measurement of rest frame $\mathrm{V}$ and $\mathrm{B}$ band magnitude to $2 \%$ near peak luminosity.

- Sufficient filters to perform an accurate K-correction.

- Measurement of B-V color evolution.

- Sufficiently early detection of SNe explosion time to eliminate detection bias.

- Measurement of multiple points on the rising side of the light curve.

- Measurement of light curve "stretch."

- A measurement of the peak-to-tail luminosity ratio.

- Determination of the host-galaxy photometric redshift. 
Imager detector specifications are given in Table 1.

Table 1. Mission reference specifications for the imager and its sensors.

\begin{tabular}{|l|c|c|c|}
\hline Parameter & Visible & NIR & Units \\
\hline FOV & 0.34 & 0.34 & $\mathrm{deg}^{2}$ \\
\hline Plate scale (nominal) & 0.10 & 0.17 & $\mathrm{arcsec}$ \\
\hline Wavelength & $350-1000$ & $900-1700$ & $\mathrm{~nm}$ \\
\hline$<$ Quantum efficiency $>$ & 80 & 60 & $\%$ \\
\hline Read noise (multiple reads) & 4 & 5 & $\mathrm{e}$ \\
\hline Dark current & 0.002 & 0.02 & $\mathrm{e} / \mathrm{s} / \mathrm{pixel}$ \\
\hline Filters & 6 & 3 & \\
\hline
\end{tabular}

The SNAP imager addresses the above requirements using two detector technologies to efficiently cover the wavelength range of $350 \mathrm{~nm}$ to $1700 \mathrm{~nm}$. The visible region $(350 \mathrm{~nm}$ to $1000 \mathrm{~nm})$ is measured with CCDs. The NIR range $(900 \mathrm{~nm}$ to $1700 \mathrm{~nm})$ is measured with $\mathrm{HgCdTe}$ devices. The $\mathrm{HgCdTe}$ devices have a fixed pixel size of $18 \mu \mathrm{m}$ and the telescope optics are designed to give an angular pixel size of $0.17 \operatorname{arcsec}$, a good match to the telescope diffraction limit at $1700 \mathrm{~nm}[6]$. We have selected $10.5 \mathrm{~mm}$ pixels for the CCDs to provide better sampling of the reduced Airy disk in the visible region. Here the angular pixel size is 0.10 arcsec.

\subsection{Visible detectors}

The main area of development for visible detectors is the LBNL CCD technology[7,8,9]. These CCDs are implemented on high resistivity silicon with p-channels and operated fully-depleted. The nominal thickness we are targeting is $200 \mu \mathrm{m}$; this is a compromise between extended red response, minimizing cosmic ray impact, and minimizing diffusion. Figure 7 shows the quantum efficiency of an LBNL CCD[10]. The good QE above $800 \mathrm{~nm}$ is apparent. The use of high purity, n-type silicon has proven to be very radiation tolerant in the accelerator environment of high energy physics experiments. Early measurements of the CCD charge transfer efficiency degradation as a function of proton dose indicates that these CCDs will be more radiation tolerant than previously flown devices[11,12]. Further characteristics of this CCD technology are presented elsewhere at this conference[13].

One unique feature of the back-illuminated and over-depleted LBNL CCDs is that the charge diffusion of holes generated at the backside can be controlled. Even though charge has to drift through several hundred microns of substrate to the pixel wells, the point spread function can be well controlled

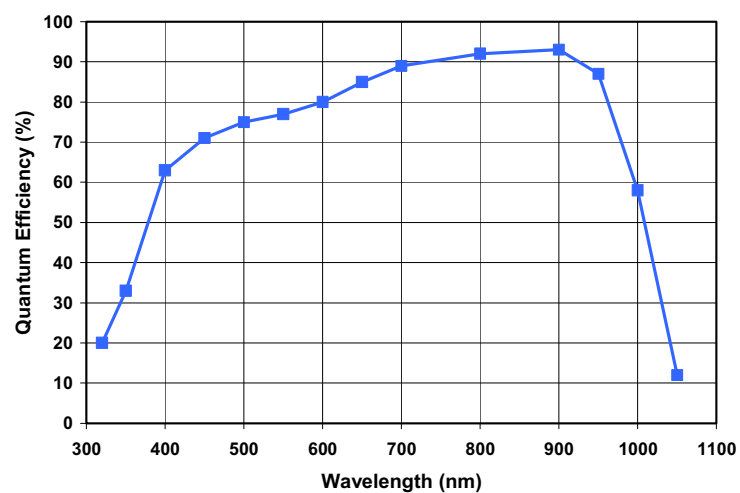

Figure 7. Quantum efficiency for a $300 \mu \mathrm{m}$ thick LBNL CCD with a simple InTiO/ $/ \mathrm{SiO}_{2}$ AR coating. with the substrate voltage, see Figure 8 and Figure 9.

Charge diffusion must be well controlled in SNAP CCDs due to the relatively small plate scale; this is one of the main drivers for $10.5 \mu \mathrm{m}$ pixels. To make effective use of this pixel size, we require the diffusion to be of order $4 \mu \mathrm{m}$. Figure 9 shows $6 \mu \mathrm{m}$ rms diffusion at $80 \mathrm{~V}$ for a $300 \mu \mathrm{m}$ device; this will scale to $4 \mu \mathrm{m}$ for a $200 \mu \mathrm{m}$ thick device.

LBNL CCDs are presently fabricated in a two-step process beginning with commercial fabrication on $150 \mathrm{~mm}$ wafers of all polysilicon, oxide, and implant steps. Backside processing and metallization occur at LBNL. A wafer design containing four SNAP format CCDs is presently in fabrication. The devices are $3512 \times 3512,10.5 \mu \mathrm{m}$ pixel with 4corner readout. The devices will be finished to a thickness of $200 \mu \mathrm{m}$. The design incorporates techniques to insure robust operation at high depletion voltage in a radiation environment. 
As an alternative to CCDs, we are performing an evaluation of the hybridized silicon PIN photodiodes. Such parts are available from Rockwell (HyVisi) and Raytheon[14]. Issues to be studied for this technology are dark current and spatial resolution. Additionally, the pixel size of these parts, $18-20 \mu \mathrm{m}$, is too large for the diffraction scale of the SNAP telescope at visible wavelengths. To be useful for SNAP, these devices must be redesigned for smaller pixel sizes.
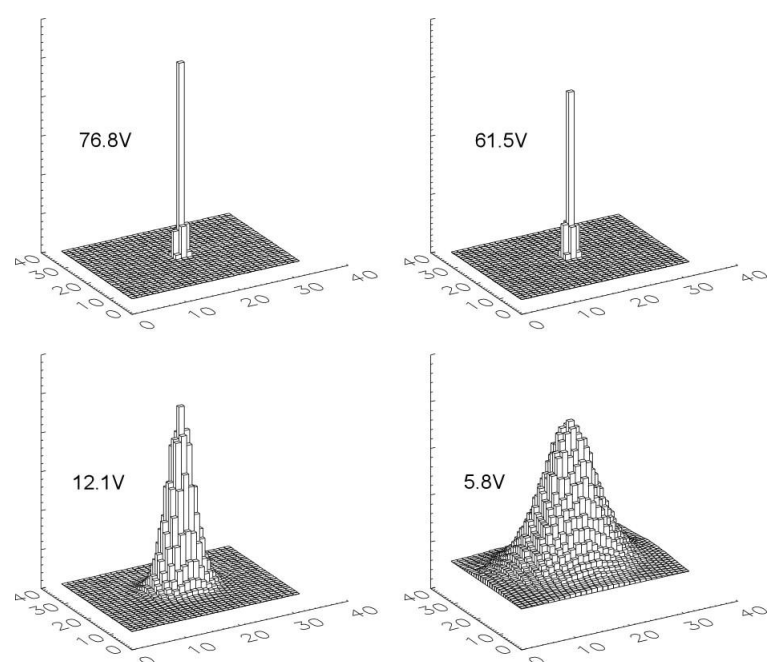

Figure 8. A $1 \mu \mathrm{m}$ rms pinhole is projected onto a backilluminated $300 \mu \mathrm{m}$ thick, $15 \mu \mathrm{m}$ pixel LBNL CCD. The panels correspond to various values of the depletion voltage.

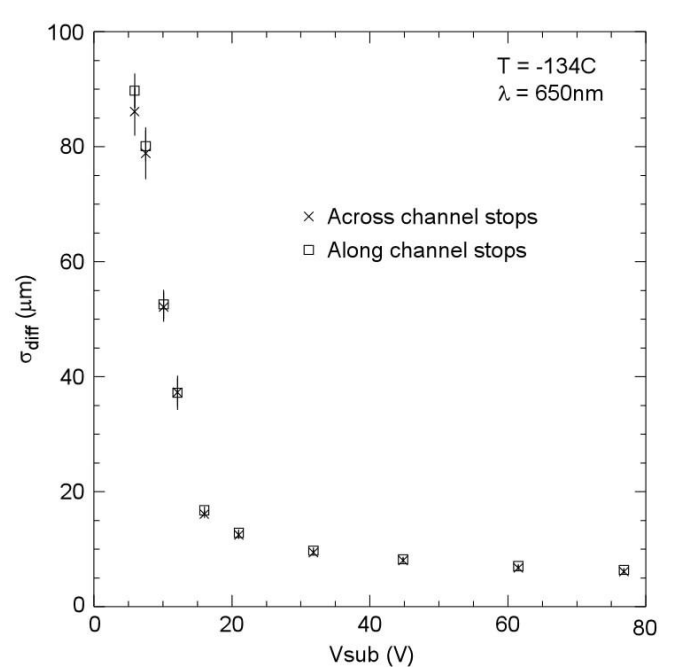

Figure 9. Plotted are results of the rms diffusion value achieved as a function of the depletion voltage. Below $20 \mathrm{~V}$, the device is under-depleted operation. Above $20 \mathrm{~V}$, an expected $\mathrm{V}^{-1 / 2}$ scaling is observed.

\subsection{Near-infrared detectors}

NIR detectors with the $1.7 \mu \mathrm{m}$ cutoff desired by SNAP have been developed by Rockwell Science Center for the HST Wide Field Camera 3[15]. SNAP has contracted Rockwell to extend this technology to $2 \mathrm{k}$ x $2 \mathrm{k}$ format devices on the newest generation of readout multiplexers developed for the JWST[16]. The photoactive material is HgCdTe PIN diodes with the starter material grown by molecular beam epitaxy. The pixel size is $18 \mu \mathrm{m}$.

SNAP also contracted Raytheon Vision Systems to develop $1.7 \mu \mathrm{m}$ cutoff material grown by liquid phase epitaxy. Development devices will be $1 \mathrm{k}$ x $1 \mathrm{k}$ format. This will lead to $2 \mathrm{k} \times 2 \mathrm{k}$ format devices hybridized to the newest readout multiplexer developed for the $2.5 \mu \mathrm{m}$ cutoff detectors being fabricated for VISTA[17]. In both cases, the pixel size is $20 \mu \mathrm{m}$.

An interesting material with bandpass matching the needs of SNAP is $\mathrm{In}_{53} \mathrm{Ga}_{47} \mathrm{As}$ used extensively in fiber optics communications. The $\mathrm{QE}$ in high radiance, room temperature use is high from $900 \mathrm{~nm}$ to $1.7 \mu \mathrm{m}$. To date, we have verified that the high wavelength cutoff decreases by $8 \AA / \mathrm{K}$ and that the dark current for a large area photodiode scales with temperature approximately as expected. To make further progress, we plan to contract the fabrication of a modest sized array, hybridized onto an astronomy-grade readout multiplexer.

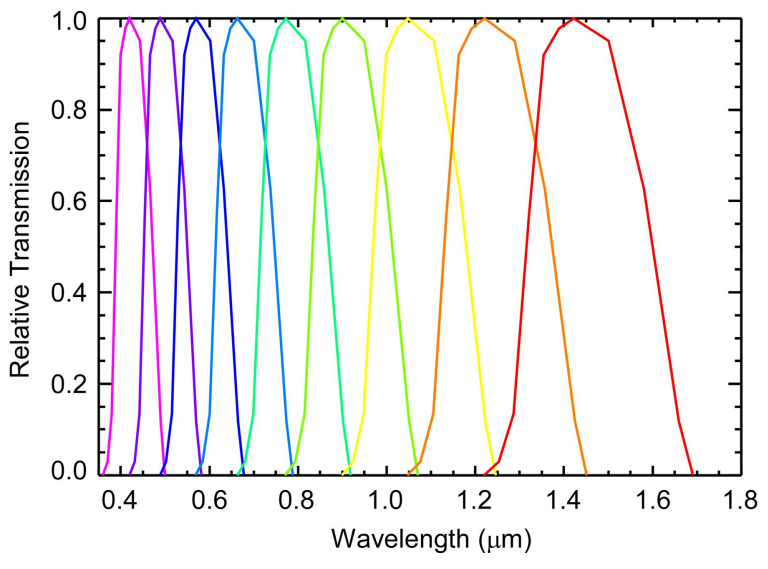

Figure 10. The SNAP filter set is defined simply as a B-band filter wavelength scaled according to $1.16^{\mathrm{n}}$. 


\subsection{Filters}

The issues defining a filter set for SNAP are photo-z determination, color, and K-correction. The latter is the primary driver. Simply, this is the reconstruction of the SNe restframe flux or flux ratios from a set of laboratory-frame filter measurements. The application of K-corrections to supernova spectra is well established[18]. A study was performed to determine the minimum filter set required[19]. We find that six visible filters and three NIR filters are sufficient if they are derived from a Johnson B-band filter with $1.16^{\mathrm{n}}$ scaling for $\mathrm{n}=0$ to 8 as shown in Figure 10 .

To implement the filters, we are investigating the direct deposition of interference filters on the sensors. We have started this program with CCDs; progress is reported elsewhere at this conference[20]. Issues are deposition damage to the fragile CCD back side and creation of multiple filters on one substrate in the case where two filter-types are required per $\mathrm{CCD}$.

\section{SPECTROGRAPH}

The spectroscopy requirements are (refer to Figure 2):

- Measurement of UV metallicity features with a S/N of 20 .

- Identify SII feature at $5350 \AA$, line width of $200 \AA$.

- Identify SII "W" shape, line width of $75 \AA$.

- Identify SiII feature at $6150 \AA$, line width of $200 \AA$.

- Measure a collection of ejecta features with fitted resolution of $15 \AA$.

- Measure host galaxy redshift.

- Provide accurate spectrophotometric calibration of the imager.

From these requirements, the derived instrument specifications are shown in Table 2. An integral field unit is used, implemented as an image slicer, to reduce the absolute pointing requirements of the satellite and to allow simultaneous measurement of the host galaxy spectra. Following this is a two-channel spectrometer to cover the wavelength range[21].

Table 2. Mission reference specifications for the spectrograph and its sensors.

\begin{tabular}{|l|c|c|c|}
\hline Parameter & Visible & NIR & Units \\
\hline Wavelength coverage & $350-980$ & $980-1700$ & $\mathrm{~nm}$ \\
\hline Plate scale & 0.15 & 0.15 & $\operatorname{arcsec}$ \\
\hline Spatial resolution & 0.15 & 0.15 & $\operatorname{arcsec}$ \\
\hline Field-of-View & $3 \times 6$ & $3 \times 6$ & $\operatorname{arcsec}^{2}$ \\
\hline Resolution & $200-70$ & $100-70$ & $\lambda / \delta \lambda$ \\
\hline$<$ Quantum Efficiency $>$ & 80 & 60 & $\%$ \\
\hline Read Noise & 2 & 5 & $\mathrm{e}$ \\
\hline Dark Current & 0.001 & 0.02 & $\mathrm{e} / \mathrm{s} / \mathrm{pixel}$ \\
\hline
\end{tabular}

The image slicer technique is used in order to eliminate slit loss for spectrophotometry. Figure 11 shows the principle of this technique. The field of view is sliced along 4 strips on a "slicing mirror". Each slice re-images the telescope pupil and a tilt in each slice reformats the 4 pupil images into a line forming a "pseudo-slit." The pseudo-slit is placed in the entrance plane of the spectrograph, acting as the entrance slit. For SNAP, the slicer stack will contain 20 elements. 


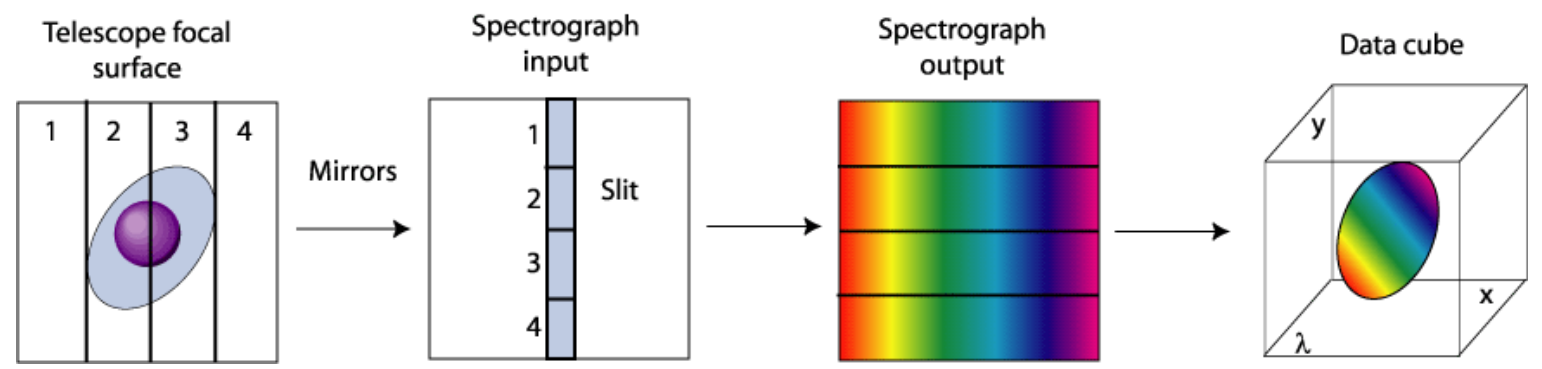

Figure 11. The slicer unit acts as a field reformatting system. As described above, the principle is to slice a 2D field of view into long strips and optically align all the strips to form a long spectrograph entrance slit.

Prisms are used as the spectrograph dispersive elements because of their high throughput and SNAP's modest resolution requirements (see Figure 13). BK7 is used for the visible channel with a dichroic on one surface to transmit the NIR to a CaF2 prism. A redundant pair of CCDs and $\mathrm{HgCdTe}$ detectors are used in the visible and NIR channels, respectively. Simulations of the efficiency of the instrument show very good results, $53 \%$ in the visible and $35 \%$ in the NIR. The principal losses are from the prisms and detectors.

A slicer prototype is under construction and test a facility for evaluating detectors is being established.

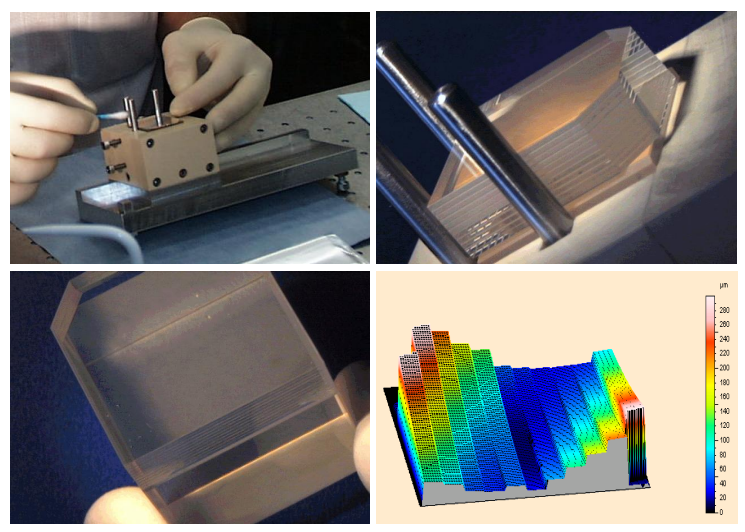

Figure 12. The slicing mirror is comprised of a stack of slicers (top right). Each slicer has an optically active spherical surface on one edge (bottom right).

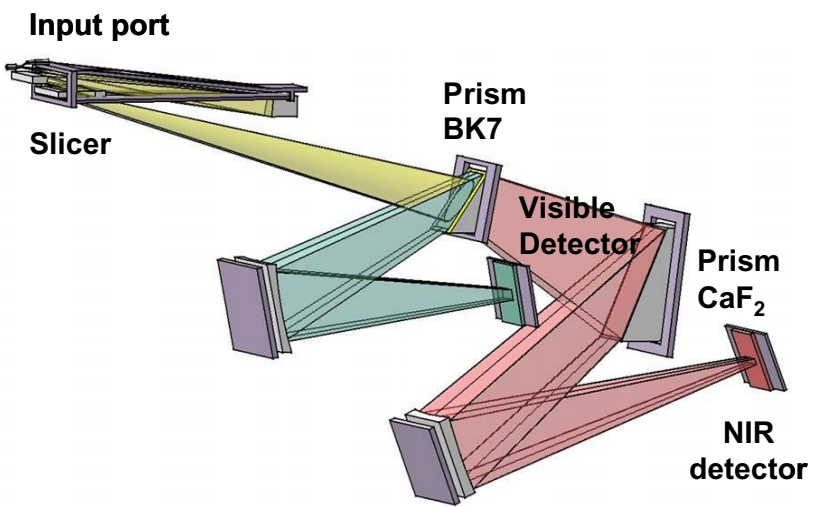

Figure 13. Optical path and elements of an image slicer IFU spectrograph with split optical and NIR dispersive channels.

\section{STAR GUIDER}

We are considering the placement of specialized sensors on the focal plane to provide near-video-rate star guiding information to the spacecraft attitude control system during exposures. An initial study indicated that there are sufficient stars brighter than V=16 such that four $1 \mathrm{k}$ x $1 \mathrm{k}$ CCDs $\left(200 \times 200 \operatorname{arcsec}^{2}\right)$ would provide a $95 \%$ probability of finding a guide star[22]. Further study has since found that R-band magnitudes are more appropriate for silicon detectors, 95\% probability is not sufficient, and that the sky region for SNAP surveys is NGP, not NEP[23]. To achieve $99 \%$ success for acquiring a single guide star requires $161 \mathrm{k} \mathrm{x} 1 \mathrm{k}$ CCDs at 30 frames per second. Lower frame rates, i.e. longer integration times for dimmer stars, might be possible with higher quantum efficiency, large bandwidth detectors such as LBNL CCDs or Rockwell HyVisi silicon hybridized PIN photodiodes. We also note that Rockwell visible or NIR detectors managed by the SIDECAR ASIC allow high read-rate of regions of interest in an array without disturbing science data. This leads to the possibility of using the imager itself, either visible or NIR, to provide star guiding information without the introduction of another system onto the focal plane. Detector evaluation and operating mode, development of an appropriate centroiding and Kalman filter algorithms, and interaction with the spacecraft attitude control system are research areas during the next two years. 


\section{READOUT ELECTRONICS}

We are exploring the photons-to-bits paradigm for both visible and NIR sensors. The goal is to digitize detector analog signals as close as possible to the detectors. The motivation is mitigating system issues that might only become apparent at satellite integration time, such as pick-up on cables carrying $\mu \mathrm{V}$ signals. We desire to go even further and incorporate device readout control near the detectors to minimize cabling and to provide a highly partitioned system with fine granularity in its failure modes.

An example of a fully integrated detector controller is the Rockwell SIDECAR[24], presently under development. This ASIC generates control signals and biases to the detector readout multiplexer, digitizes up to 32 outputs at 16-bits, and provides multiple serial data output streams. Since the NIR detectors will require multiple reads to reduce the read noise, SNAP would need to operate the SIDECAR in 32-channel mode at approximately $100 \mathrm{kpixel} / \mathrm{s}$. This ASIC can be co-located with the cold detector.

A SIDECAR-like solution for CCDs is more challenging because of the large range of voltages required to operate them. In the case of the LBNL CCD, the required voltage range is $33 \mathrm{~V}$, excluding the depletion voltage. We consider it most important to digitize the CCD analog outputs near the CCD and have developed a four channel dual-slope correlated double sampler (CDS) ASIC. The CDS chip has been prototyped in a $0.25 \mu \mathrm{m}$ CMOS technology using radiation tolerant-by-design principles. The CDS auto selects between three gain ranges; two range bits and a 12-bit precision analog level are generated. Figure 14 is a block diagram of one channel and Figure 15 shows the output of the CDS for a full scale input. The lower curve represents the integrator output in multi-range mode, showing the 3 gains switching. The upper curves show the digital output bits indicating the active gain range. A non-linearity of $\pm 0.025 \%$ and noise of $7 \mu \mathrm{V}$ rms has been achieved in the first fabricated devices. On the most sensitive range, the CDS will not degrade the LBNL CCD read noise of 2(4) e at 50(100) kpixel/s. The next advancement of this ASIC will be the incorporation of a 12-bit pipeline ADC for each CDS channel. With a measured power consumption of $6.3 \mathrm{~mW}$ for the CDS and an overall budget of $10 \mathrm{~mW}$ per channel, the final ASIC incorporating the ADC can be co-located with the cold CCD. We are also exploring the integration of the CCD clocking and bias voltage generation in a radiation tolerant ASIC.

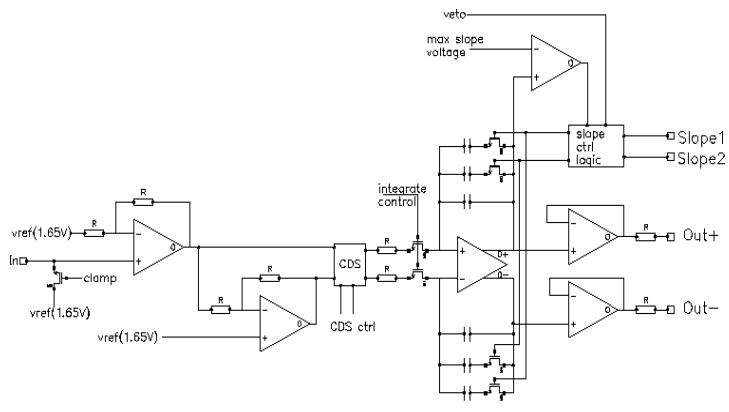

Figure 14. Block diagram for a three range dual slope correlated double sampler implemented as an ASIC in 0.25 $\mu \mathrm{m}$ CMOS. The selection of capacitors in the feedback loop set the gains to 32,2 , and 1 .

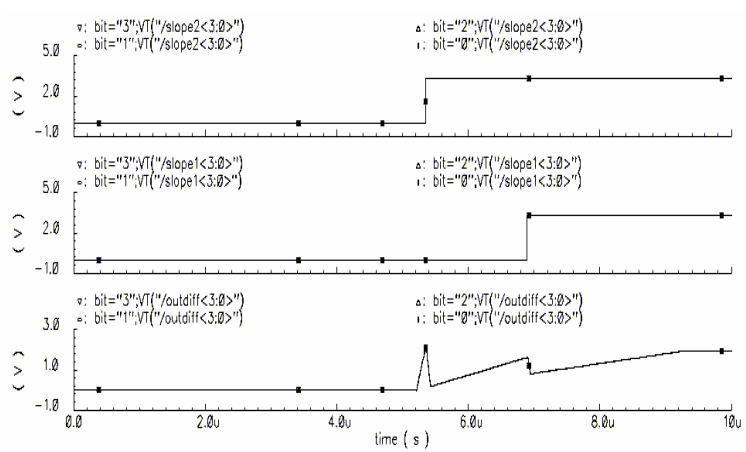

Figure 15. Simulated waveforms for the CDS with a full scale input. The transitions in the bottom waveform correspond to range shifting to progressively less sensitive ranges and are recorded in the top two digital traces. The analog signal is digitized at the last time marker on the right.

\section{CONCLUSION}

The SNAP instrument focal plane concept and operation is mature. We are now concentrating on a two year R\&D period to bring a few key technologies to sufficient maturity in preparation for a mission proposal. These include 
LBNL CCDs and hybridized silicon PIN photodiodes for visible detectors, HgCdTe and InGaAs hybridized detectors for the NIR, direct deposition of filters on detectors, ASIC solutions for detector control and data conversion, spectrograph image slicer prototype, and on-focal-plane star guide strategy.

\section{ACKNOWLEDGMENTS}

This work was supported by the Director, Office of Science, of the U.S. Department of Energy under Contract No. DE-AC03-76SF00098.

\section{REFERENCES}

1. G. Aldering et al., "Overview of the SuperNova/Acceleration Probe (SNAP)," Proc. SPIE 4835-146, 2002.

2. S. Perlmutter et al., "Omega and Lambda from 42 High-Redshift Supernovae," ApJ 517, p.565, 1999.

3. A. G. Riess et al., "Observational Evidence from Supernovae for an Accelerating Universe and a Cosmological Constant," AJ, 116, p.1009. 1998.

4. M. Lampton et al., "SNAP Telescope," Proc. SPIE 4849-215, 2002.

5. M. Lampton et al., "SNAP focal plane," Proc. SPIE 4854-632, 2002.

6. M. Lampton et al., "SNAP telescope," SPIE conf. 5166 talk 12, 2003.

7. S. E. Holland et al., "A 200 x 200 CCD Image Sensor Fabricated on High-Resistivity Silicon," IEDM Tech. Digest, 911 (1996).

8. S. E. Holland, N. W. Wang, and W. W. Moses, "Development of low noise, back-side illuminated silicon photodiode arrays," IEEE Trans. Nucl. Sci. 44, 443-447 (June 1997).

9. S. E. Holland, D. E. Groom, N. P. Palaio, R. J. Stover, and M. Wei, "Fully Depleted, Back-Illuminated ChargeCoupled Devices Fabricated on High-resistivity Silicon," IEEE Trans. Electron Devices, 50, pp. 225-238, Jan 2003.

10. J. Beletic, R. Stover, K. Taylor, "An assessment of the optical detector systems of the W.M. Keck Observatory," private communication, 19 January 2001.

11. C. J. Bebek et al., "Proton radiation damage in high-resistivity n-type silicon CCDs," SPIE 4669-161, 2002.

12. C. J. Bebek et al., "Proton radiation damage in p-channel CCDs fabricated on high-resistivity silicon," IEEE Trans. Nucl. Sci. vol. 49, pp. 1221 -1225, Jun. 2002.

13. S.E. Holland et al., "Fully depleted back-illuminated p-channel CCD development," SPIE conf. 5167 talk 07, 2003.

14. A.C. Moore et al., "Operation and Test of Hybridized Silicon p-i-n Arrays Using Open-source Array Control Hardware and Software," to be published, 19 December 2002.

15. M. Robberto et al., "Infrared Detectors for WFC3 on the Hubble Space Telescope," preprint, August 2002 (Hawaii).

16. M. Loose et al., Proc. SPIE 4850-867, 2003.

17. A.M. McPherson, "Project VISTA: a review of its progress and overview of the current program," Proc. SPIE 4837-82, 2003.

18. P. Nugent, A. Kim, and S. Perlmutter, "K-Corrections and Extinction Corrections for Type Ia Supernovae," PASP, $114,803 \mathrm{~N}(2002)$.

19. A. Kim, "K-correction Errors", private communication, 2003.

20. C. Bower, "SNAP spectral filters," SPIE conf. 5167 talk 05, 2003.

21. A. Ealet et al., "SNAP: an integral field spectrograph for supernova identification," Proc. SPIE 4850-1169, 2002.

22. A. Secroun et al., Experimental Astron. 12, p. 69, 2001.

23. http://albert.ssl.berkeley.edu/ mlampton/.

24. M. Loose et al., Proc. SPIE 4841-782, 2003.

*cjbebek@lbl.gov; phone 1510 486-6447; fax 1 510 486-7115 